\title{
UPDATE ON THE WAITING LIST REDUCTION PROGRAM
}

Nick Shiraev and Jean McGarry Evaluation and Monitoring Branch Performance Management Division NSW Health Department

The October issue of the Public Health Bulletin contained an article describing the initial results of the Waiting List Reduction Program to the end of September 1995'. This report updates the results to the end of October.

The Program was introduced to improve access to elective surgery services in public hospitals throughout NSW. The aim is to reduce the March 31, 1995 elective surgery waiting lists by 50 per cent within 12 months, concentrating on people who have been waiting more than six months for surgery.

\section{RESULTS}

By October 31 the elective surgery waiting list had decreased from 44,707 to 29,780 patients, a reduction of 14,927 patients (33 per cent) (Table 5).

Since March 31 the number of people waiting longer than 12 months has declined by 1,343 ( 59 per cent), and the number waiting between six and 12 months has fallen by 3,674 (58 per cent) (Table 6). The waiting list has already been halved for people waiting more than six months; the most significant effect of the Program has been the reduction in the number of people waiting more than six months by over 5,000 .

Particularly significant were reductions in numbers of people waiting longer than six months for procedures such as cholecystectomy (down by 207 or 70 per cent), cataract extraction (down by 771 or 63 per cent), tonsillectomy (down by 416 or 67 per cent) varicose vein stripping and ligation (down by 303 or 72 per cent) and total hip replacement (down by 78 or 43 per cent).

The average waiting time of patients treated in the course of a month rose from 1.4 months in March to 1.7 months in October. This reflects large numbers of admissions of patients who have been waiting long periods. The clearance time has reduced to 1.4 months from 1.8 months, indicating patients have been admitted more quickly. The average time on the list has fallen from 3.6 months to 2.8 months, indicating patients are waiting on the list almost a month less (Table 7). This overall pattern is reflected in most Areas and Districts (Table 7).

In the period from July 1, 1994 to March 31, 1995 admissions increased by 2.5 per cent compared with the corresponding period a year previously. Then between April

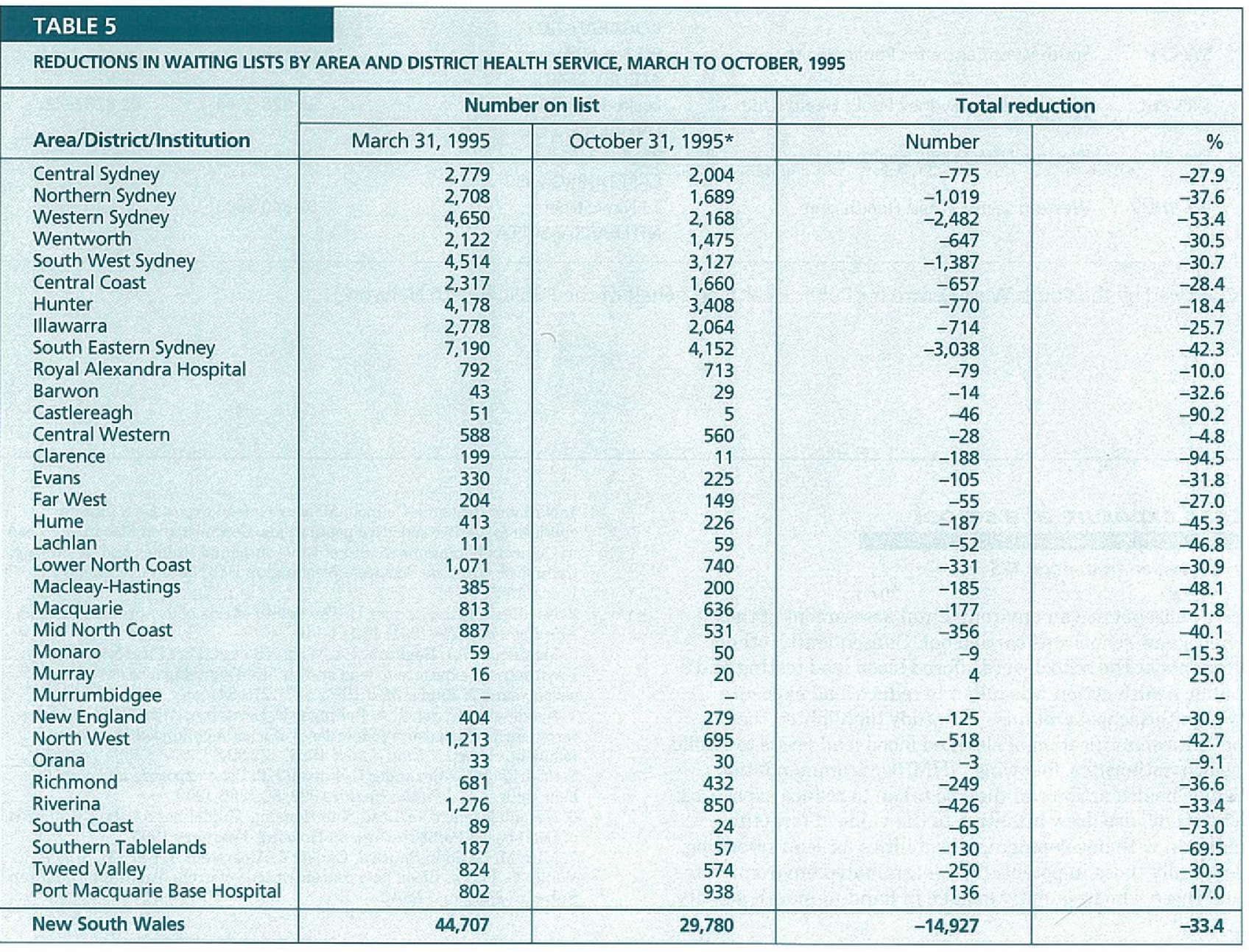

Source: Department of Health Reporting System, November 9, 1995

* Excludes transfers. List transfers, in general, are an administrative change and not a change in local demand.

They are therefore not taken into account when estimating changes in the number of patients on a list. 
and October 1995, the total number of admissions to hospitals in NSW rose by 35,744 - or 4.8 per cent compared with the corresponding period in 1994. On an annual basis this is equivalent to more than 60,000 extra admissions. Thus the increase in admissions attributable to the Waiting List Reduction Program to October 31, 1995 is estimated to have been 2.3 per cent, representing 28,000 admissions on an annual basis.

It is also estimated that, to October 31,1995 , at least 16,386 extra procedures were performed as part of the Waiting List Reduction Program. This is likely to be an underestimate because, in mid-1994, a large number of medical procedures, such as endoscopies and chemotherapy, was erroneously coded as surgery. Most of the increase occurred in the period June-October 1995, but there was some minor increase in April and May. The rate of increase of elective surgery was 11.2 per cent, more than double the general increase (total all programs) of 4.8 per cent in the same period (Table 8 ).

The changes in listing status over the past five quarters are shown in Figure 2. The main reason for removal of patients from waiting lists was that the treatment was no longer

\section{FIGURE 2}

LISTING STATUS CHANGES FROM READY FOR CARE (EXCLUDING ADMISSIONS)

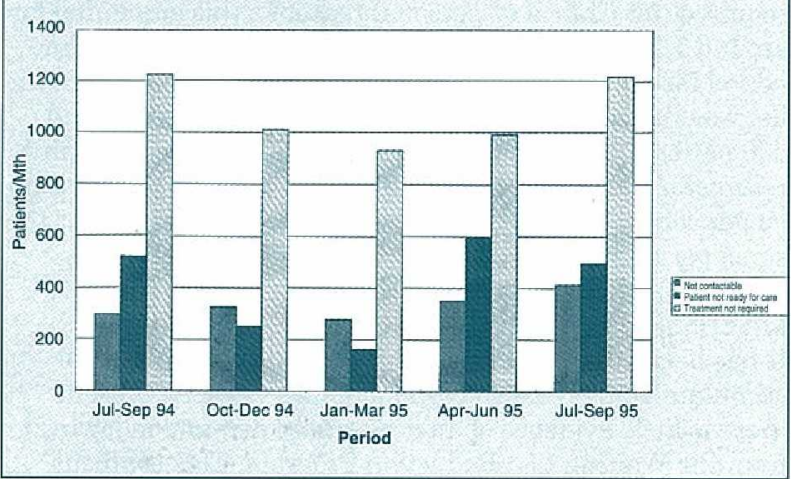

\section{TABLE 6}

REDUCTIONS IN PATIENTS ON AREA AND DISTRICT WAITING LISTS, OCTOBER, 1995 PATIENTS WAITING LONGER THAN SIX MONTHS

\begin{tabular}{|c|c|c|c|c|c|c|c|c|}
\hline \multirow[b]{4}{*}{ Area/District/Institution } & \multicolumn{8}{|c|}{ NUMBER ON LIST } \\
\hline & \multicolumn{4}{|c|}{ Waiting 6-12 months } & \multicolumn{4}{|c|}{ Waiting > 12 months } \\
\hline & \multirow[b]{2}{*}{ Mar 31, 1995} & \multirow[b]{2}{*}{ Oct 31, 1995} & \multicolumn{2}{|c|}{ Reduction } & \multirow[b]{2}{*}{ Mar 31, 1995} & \multirow[b]{2}{*}{ Oct 31,1995} & \multicolumn{2}{|c|}{ Reduction } \\
\hline & & & Number & $\%$ & & & Number & $\%$ \\
\hline Central Sydney & 116 & 19 & -97 & -84 & 15 & 0 & -15 & -100 \\
\hline Northern Sydney & 404 & 107 & -297 & -74 & 173 & 100 & -73 & -42 \\
\hline Western Sydney & 836 & 139 & -697 & -83 & 295 & 74 & -221 & -75 \\
\hline Wentworth & 340 & 125 & -215 & -63 & 293 & 134 & -159 & -54 \\
\hline South West Sydney & 555 & 309 & -246 & -44 & 191 & 143 & -48 & -25 \\
\hline Central Coast & 398 & 254 & -144 & -36 & 107 & 25 & -82 & -77 \\
\hline Hunter & 768 & 415 & -353 & -46 & 179 & 94 & -85 & -47 \\
\hline Illawarra & 463 & 181 & -282 & -61 & 173 & 74 & -99 & -57 \\
\hline South Eastern Sydney & 1,150 & 467 & -683 & -59 & 559 & 161 & -398 & -71 \\
\hline Royal Alexandra Hospital & 25 & 8 & -17 & -68 & 11 & - & -11 & -100 \\
\hline Barwon & - & - & - & - & - & - & - & -100 \\
\hline Castlereagh & - & - & - & - & - & - & - & - \\
\hline Central Western & 80 & 57 & -23 & -29 & 8 & 8 & - & - \\
\hline Clarence & 1 & - & -1 & -100 & - & - & - & 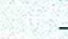 \\
\hline Evans & 2 & - & -2 & -100 & - & - & - & - \\
\hline Far West & $\overline{1}$ & - & -1 & -100 & - & - & - & - \\
\hline Hume & 25 & 14 & -11 & -44 & 4 & - & -4 & -100 \\
\hline Lachlan & 13 & - & -13 & -100 & - & - & - & -100 \\
\hline Lower North Coast & 207 & 146 & -61 & -29 & 52 & 1 & -51 & -98 \\
\hline Macleay-Hastings & 101 & 10 & -91 & -90 & 46 & 6 & -40 & -87 \\
\hline Macquarie & 164 & 58 & -106 & -65 & 41 & 42 & 1 & 2 \\
\hline Mid North Coast & 150 & 31 & -119 & -79 & 4 & 1 & -3 & -75 \\
\hline Monaro & - & - & - & - & - & - & - & 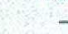 \\
\hline Murray & - & - & - & - & - & - & - & - \\
\hline Murrumbidgee & - & - & - & - & - & - & - & - \\
\hline New England & 26 & - & -26 & -100 & 1 & - & -1 & -100 \\
\hline North West & 169 & 61 & -108 & -64 & 16 & 2 & -14 & -88 \\
\hline Orana & 4 & 1 & -3 & -75 & - & 2 & 2 & - \\
\hline Richmond & 95 & 31 & -64 & -67 & 1 & 4 & 3 & 300 \\
\hline Riverina & 199 & 82 & -117 & -59 & 77 & 5 & -72 & -94 \\
\hline South Coast & 4 & 2 & -2 & -50 & - & - & $-1<$ & -3 \\
\hline Southern Tablelands & 1 & 1 & - & - & - & - & - & 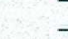 \\
\hline Tweed Valley & 79 & 20 & -59 & -75 & 19 & - & -19 & -100 \\
\hline Port Macquarie Base Hosp & 3 & 167 & 164 & 5,467 & - & 46 & 46 & - \\
\hline New South Wales & 6,379 & 2,705 & $-3,674$ & -58 & 2,265 & 922 & $-1,343$ & -59 \\
\hline
\end{tabular}

Source: Department of Health Reporting System, November 9, 1995 


\section{Update on waiting lists}

\section{$\checkmark$ Continued from page 129}

required (for clinical or personal reasons); this accounted for around 1,200 patients, virtually unchanged from the same period last year. The next major reason for removal from the list was that the patients were not ready for care (around 500 patients a month, again similar to last year). The third reason for removal from the list was that the patient was no longer contactable. This has increased by about 100 over the same period last year.

\section{DISCUSSION}

It has been suggested that reductions in waiting lists stimulate an increase in demand for services. At present there is little evidence of an accelerated demand following from the Waiting List Reduction Program. This confirms recent overseas findings ${ }^{2}$ that induced demand resulting from substantial reductions in waiting lists is, at least in the short term, rather small. It will be interesting to see whether a surge in demand is apparent early in 1996.
Predicted changes observed in the flow patterns are the result of growing and relatively under-resourced areas being able to provide more of their residents with services locally. Examples are the Wentworth and Central Coast Areas.

It is now appropriate to direct the focus of attention to waiting times and the achievement of best practice in the management of elective patients. Future issues for consideration may include development of benchmarks for:

\begin{tabular}{l|l} 
patients allocated clinical urgency codes of 1 \\
(should be admitted within seven days) or \\
2 (should be admitted within one month); \\
theatre utilisation; \\
admissions delayed by hospital constraints; and \\
same day surgery.
\end{tabular}

1. Shiraev N, McGarry J. Waiting List Reduction Program: initial results. NSW Public Health Bulletin 1995; 6(10):106-109.

2. Martin S, Smith P. Modelling waiting times for elective surgery. Centre for Health Economics, The University of York, 1995.

\begin{tabular}{|c|c|c|c|c|c|c|c|c|c|}
\hline \multirow[b]{3}{*}{ Area/District/Institution } & \multicolumn{9}{|c|}{ WAITING TIMES (Months) } \\
\hline & \multicolumn{3}{|c|}{ Expected waiting time } & \multicolumn{3}{|c|}{ Average waiting time } & \multicolumn{3}{|c|}{ Average time on list } \\
\hline & Mar 31, 95 & Oct31, 95 & Variation* & Mar 31,95 & Oct 31,95 & Variation** & Mar 31, 95 & Oct 31,95 & Variation* \\
\hline Central Sydney & 1.1 & 0.9 & -0.3 & 1.0 & 0.9 & 0.1 & 1.5 & 1.0 & -0.5 \\
\hline Northern Sydney & 1.3 & 1.0 & -0.3 & 0.9 & 2.3 & 1.4 & 3.5 & 2.8 & -0.7 \\
\hline Western Sydney & 1.9 & 1.3 & -0.6 & 1.6 & 1.5 & 0.0 & 4.1 & 2.6 & -1.5 \\
\hline Wentworth & 2.6 & 1.7 & -0.9 & 2.1 & 2.6 & 0.4 & 5.9 & 4.4 & -1.4 \\
\hline South West Sydney & 1.7 & 1.5 & -0.2 & 1.4 & 1.6 & 0.2 & 3.3 & 3.4 & 0.2 \\
\hline Central Coast & 2.5 & 2.1 & -0.4 & 1.9 & 2.2 & 0.3 & 4.0 & 3.0 & -1.0 \\
\hline Hunter & 2.0 & 1.8 & -0.2 & 1.6 & 1.8 & 0.2 & 3.7 & 3.1 & -0.6 \\
\hline Illawarra & 2.4 & 1.7 & -0.6 & 2.3 & 2.3 & 0.0 & 3.9 & 2.9 & -1.1 \\
\hline South Eastern Sydney & 1.7 & 1.4 & -0.3 & 1.2 & 1.5 & 0.2 & 4.2 & 3.2 & -1.0 \\
\hline Royal Alexandra Hospital & 1.3 & 2.3 & 0.9 & 1.3 & 1.0 & -0.3 & 1.6 & 1.4 & -0.2 \\
\hline Barwon & 0.6 & 0.4 & -0.2 & 0.5 & 0.4 & 0.0 & 0.7 & 0.3 & -0.4 \\
\hline Castlereagh & 0.6 & 0.4 & -0.2 & 0.5 & 0.8 & 0.3 & 0.5 & 3.1 & 2.6 \\
\hline Central Western & 1.4 & 1.4 & 0.0 & 1.1 & 1.7 & 0.6 & 2.6 & 2.7 & 0.1 \\
\hline Clarence & 1.6 & 0.5 & -1.1 & 0.4 & 0.4 & 0.0 & 1.9 & 0.4 & -1.5 \\
\hline Evans & 0.9 & 0.8 & -0.1 & 0.8 & 0.8 & 0.0 & 1.1 & 1.0 & -0.1 \\
\hline Far West & 1.4 & 1.1 & -0.2 & 0.9 & 1.1 & 0.2 & 1.4 & 1.1 & -0.3 \\
\hline Hume & 0.8 & 0.8 & 0.0 & 0.9 & 0.7 & -0.2 & 2.0 & 1.9 & -0.2 \\
\hline Lachlan & 0.9 & 0.5 & -0.4 & 0.3 & 0.7 & 0.3 & 2.1 & 1.4 & -0.8 \\
\hline Lower North Coast & 5.4 & 2.5 & -2.9 & 3.8 & 4.3 & 0.5 & 3.8 & 3.2 & -0.7 \\
\hline Macleay-Hastings & 2.2 & 1.3 & -0.9 & 2.1 & 1.2 & -0.8 & 4.8 & 2.0 & -2.8 \\
\hline Macquarie & 2.6 & 2.3 & -0.3 & 1.5 & 2.1 & 0.6 & 4.0 & 3.6 & -0.4 \\
\hline Mid North Coast & 3.5 & 1.4 & -2.2 & 2.4 & 1.8 & -0.6 & 2.9 & 2.2 & -0.7 \\
\hline Monaro & 0.7 & 0.8 & 0.1 & 0.9 & 0.9 & 0.0 & 1.0 & 0.5 & -0.5 \\
\hline Murray & 0.6 & 0.7 & 0.2 & 0.4 & 0.4 & 0.0 & 0.5 & 0.4 & -0.1 \\
\hline Murrumbidgee & 0.0 & 0.7 & 0.7 & 0.0 & 0.6 & 0.6 & 0.0 & 1.0 & 1.0 \\
\hline New England & 1.5 & 1.2 & -0.4 & 1.6 & 1.0 & -0.6 & 1.9 & 0.9 & -1.0 \\
\hline North West & 2.4 & 1.8 & -0.6 & 2.0 & 2.0 & 0.0 & 3.0 & 2.3 & -0.7 \\
\hline Orana & 1.6 & 1.7 & 0.1 & 1.6 & 3.1 & 1.4 & 2.9 & 2.8 & -0.1 \\
\hline Richmond & 1.1 & 0.9 & -0.2 & 1.0 & 0.9 & -0.1 & 2.2 & 1.8 & -0.4 \\
\hline Riverina & 3.0 & 2.3 & -0.7 & 2.9 & 2.6 & -0.4 & 3.9 & 2.6 & -1.3 \\
\hline South Coast & 0.6 & 1.1 & 0.5 & 0.5 & 0.7 & 0.2 & 1.3 & 2.5 & 1.2 \\
\hline Southern Tablelands & 1.1 & 0.9 & -0.3 & 0.9 & 0.9 & 0.0 & 3.3 & 1.1 & -2.2 \\
\hline Tweed Valley & 2.9 & 1.8 & -1.1 & 1.9 & 2.2 & 0.3 & 3.0 & 1.9 & -1.1 \\
\hline Port Macquarie Base Hosp & 3.0 & 3.2 & 0.2 & 2.0 & 4.5 & 2.4 & 3.0 & 4.0 & 0.9 \\
\hline New South Wales & 1.8 & 1.4 & -0.4 & 1.4 & 1.7 & 0.3 & 3.6 & 2.8 & -0.8 \\
\hline
\end{tabular}

Source: Department of Health Reporting System, November 9, 1995

* Rounding during addition may produce minor inconsistencies in totals. 


\begin{tabular}{|c|c|c|c|c|c|c|}
\hline \multirow[b]{4}{*}{ Area/District/Institution } & \multicolumn{3}{|c|}{ ADMISSIONS - ALL PROGRAMS* } & & & \\
\hline & \multirow[b]{3}{*}{ Total } & \multirow{2}{*}{\multicolumn{2}{|c|}{ Increase }} & & & \\
\hline & & & & \multirow[b]{2}{*}{ Total } & \multicolumn{2}{|c|}{$\begin{array}{c}\text { Increase due to } \\
\text { Waiting List Reduction Program }\end{array}$} \\
\hline & & Number & $\%$ & & Number & $\%$ \\
\hline Central Sydney & 79,187 & 3,406 & 4.5 & 16,953 & 1,637 & 10.7 \\
\hline Northern Sydney & 73,958 & 2,881 & 4.1 & 13,906 & 771 & 5.9 \\
\hline Western Sydney & 76,706 & 2,969 & 4.0 & 13,863 & 1,432 & 11.5 \\
\hline Wentworth & 26,760 & 3,238 & 13.8 & 5,558 & 836 & 17.7 \\
\hline South Western Sydney & 74,123 & 7,242 & 10.8 & 16,672 & 1,494 & 9.8 \\
\hline Central Coast & 32,560 & 2,440 & 8.1 & 5,930 & 887 & 17.6 \\
\hline Hunter & 66,335 & 1,353 & 2.1 & 13,004 & 1,625 & 14.3 \\
\hline Illawarra & 37,340 & 1,503 & 4.2 & 7,366 & 699 & 10.5 \\
\hline South Eastern Sydney & 109,088 & 2,595 & 2.4 & 24,381 & 370 & 1.5 \\
\hline Royal Alexandra Hospital & 11,490 & 638 & 5.9 & 3,290 & 216 & 7.0 \\
\hline Barwon & 4,548 & -132 & -2.8 & 735 & 33 & 4.7 \\
\hline Castlereagh & 4,567 & 266 & 6.2 & 655 & 100 & 18.0 \\
\hline Central West & 10,661 & -48.5 & -4.4 & 2,068 & -551 & -21.0 \\
\hline Clarence & 5,445 & 334 & 6.5 & 684 & 172 & 33.6 \\
\hline Evans & 9,859 & 226 & 2.3 & 2,445 & 475 & 24.1 \\
\hline Far West & 4,392 & 113 & 2.6 & 973 & 214 & 28.2 \\
\hline Hume & 11,288 & 856 & 8.2 & 3,636 & 839 & 30.0 \\
\hline Lachlan & 6,168 & 485 & 8.5 & 987 & -17 & -1.7 \\
\hline Lower North Coast & 8,295 & 405 & 5.1 & 2,377 & 601 & 33.8 \\
\hline Macleay-Hastings & 10,006 & 334 & 3.5 & 2,645 & 861 & 48.3 \\
\hline Macquarie & 12,939 & 804 & 6.6 & 2,495 & 420 & 20.2 \\
\hline Mid North Coast & 9,422 & 977 & 11.6 & 1,675 & 756 & 82.3 \\
\hline Monaro & 4,047 & -139 & -3.3 & 732 & 61 & 9.1 \\
\hline Murray & 3,439 & -96 & -2.7 & 501 & -77 & -13.3 \\
\hline Murrumbidgee & 7,653 & - & - & 1,320 & 64 & 5.1 \\
\hline New England & 8,345 & 153 & 1.9 & 1,930 & 407 & 26.7 \\
\hline North West & 13,676 & 26 & 0.2 & 3,348 & 193 & 6.1 \\
\hline Orana & 3,624 & 175 & 5.1 & 114 & 5 & 4.6 \\
\hline Richmond & 20,543 & 921 & 4.7 & 4,254 & 657 & 18.3 \\
\hline Riverina & 12,211 & 528 & 4.5 & 2,878 & 374 & 14.9 \\
\hline South Coast & 8,602 & 1,101 & 14.7 & 1,643 & 433 & 35.8 \\
\hline Southern Tablelands & 7,937 & 234 & 3.0 & 1,459 & 50 & 3.5 \\
\hline Tweed Valley & 9,139 & 393 & 4.5 & 2,303 & 349 & 17.9 \\
\hline New South Wales & 784,353 & 35,744 & 4.8 & 162,780 & 16,386 & 11.2 \\
\hline
\end{tabular}

Source: Department of Health Reporting System, Waiting List Information System: Area and District returns.

* Total all admissions, all NSW public hospitals

\section{PUBLIC HEALTH EDITORIAL STAFF}

The editor of the Public Health Bulletin is Dr Michael Frommer, Director, Research and Development, NSW Health Department. Dr Lynne Madden is production manager.

The Bulletin aims to provide its readers with population health data and information to motivate effective public health action. Articles, news and comments should be 1,000 words or less in length and include a summary of the key points to be made in the first paragraph. References should be set out using the Vancouver style, the full text of which can be found in British Medical Journal 1988; 296:401-5.

Please submit items in hard copy and on diskette, preferably using WordPerfect, to the editor, NSW Public Health Bulletin, Locked Mail Bag 961, North Sydney 2059. Facsimile (02) 3919029.

Please contact your local Public Health Unit to obtain copies of the NSW Public Health Bulletin. 